\title{
NANOPARTÍCULA LIPÍDICA COM QUERCETINA NO TRATAMENTO DO CÂNCER DE PÂNCREAS: UMA REVISÃO DA LITERATURA
}

\section{Jaqueline Santana da Rosa1; Ariane Ribas Pohl2; Jefferson Potiguara de Moraes $^{2}$; Darcieli Lima Ramos²; Bianca Vedoin Copês Rambo'; Gabriéli Farencena Fernandes'; Liana da Silva Fernandes ${ }^{3}$; Virginia Cielo Rech ${ }^{4}$.}

\section{RESUMO}

Nanopartículas lipídicas vêm desempenhando um papel fundamental no tratamento anticâncer. O sucesso do emprego deste tipo de nanopartícula é devido as suas características físico-químicas, a alta biodisponibilidade, a administração por diferentes vias, capacidade de cruzar a barreira sangue-cérebro e de entregar macromoléculas como oligopeptídeos, proteínas e DNA ao alvo desejado. O objetivo dessa pesquisa foi realizar uma revisão sistemática nas bases de dados ScienceDirect, Sielo e Pubmed afim de investigar o que existe na literatura sobre tratamento de câncer de pâncreas com nanopartículas lipídicas com quercetina (NLQ). Foram utilizados os descritores "pancreatic cancer" AND nanoparticles AND "quercetin", no mês de setembro de 2021. Após a aplicação dos critérios de inclusão e exclusão, foram utilizados nessa revisão 51 artigos. Após a análise é possível concluir que atualmente, no tratamento contra o câncer de pâncreas as nanopartículas lipídicas com quercetina (NLQ) vem alcançando um lugar importante e promissor como tratamento.

Palavras-chave: Nanociência; Nanotecnologia; Neoplasia; Tumor.

Eixo Temático: Tecnologia, Inovação e Desenvolvimento Sustentável (TIDS).

\section{INTRODUÇÃO}

O câncer inicia quando as células de algum órgão ou tecido do corpo começam a crescer fora de controle. Esse crescimento é diferente do crescimento celular normal. Em vez de morrer, as células cancerosas continuam crescendo e

\footnotetext{
${ }^{1}$ Acadêmica do Curso de Biomedicina - Universidade Franciscana. jaquesantanarosa@gmail.com; biancarambo@outlook.com; gabrieli.fernandes@ufn.edu.br

2 Programa de Pós-Graduação em Nanociências - Universidade Franciscana.

ariane_pohl@yahoo.com.br; jpotiguaramoraes@yahoo.com.br; darcielilimaramos@gmail.com

3 Professora no Curso de Biomedicina - Universidade Franciscana. liana@ufn.edu.br

${ }^{4}$ Professora no Curso de Biomedicina e PPG-Nanociências - Universidade Franciscana

vga.cielo@gmail.com
} 
formando novas células anômalas. As células cancerosas também podem invadir outros tecidos (metástase), algo que as células normais não fazem. O crescimento fora de controle e a invasão de outros tecidos é o que torna uma célula cancerosa (BYUN; JUNG, 2021).

O pâncreas é uma glândula do aparelho digestivo, localizada na parte superior do abdômen e atrás do estômago. É dividido anatomicamente em três partes principais: a cabeça, corpo e cauda (MOINI, 2019). A cabeça do pâncreas está encaixada na concavidade formada pela curva do duodeno, ao lado direito do plano sagital mediano, enquanto que, a cauda está localizada à esquerda, se relacionando com o baço. A maioria dos tumores pancreáticos se desenvolve na cabeça e colo do pâncreas (NOBESCHI et al., 2012). Ele é responsável pela produção de enzimas, que atuam na digestão dos alimentos e pela insulina hormônio responsável pela redução do nível de glicose (açúcar) no sangue após as refeições (MCGUCKIN; CADE; HANISON, 2020). O câncer de pâncreas é um dos tumores que mais apresentam dificuldades para o diagnóstico precoce, geralmente, desenvolve-se de forma imperceptível, sem causar sintomas, o que torna sua detecção precoce praticamente impossível. Sua evolução é rápida e torna o câncer pancreático um dos tipos mais letais (TANG et al., 2012; SADOUGHI; MANSOURNIA; MIRHASHEMI, 2020; TRAUB; LINK; KORNMANN, 2021).

$\mathrm{Na}$ maioria dos casos é reconhecido clinicamente em estágios muito avançados. São raros antes dos 30 anos e muito comuns após os 60 anos. A incidência aumenta gradualmente conforme o avanço da idade (LEE et al., 2011; HANI et al., 2021). O câncer de pâncreas permanece por um longo período assintomático, os exames não invasivos, utilizados para detectar o câncer são: a radiografia convencional, ultrassonografia, tomografia computadorizada, ressonância magnética; enquanto que a biópsia passa a ser o método invasivo para a confirmação do câncer pancreático (NOBESCHI et al., 2012). As medidas preventivas podem ser adotadas durante a vida, como evitar o consumo de bebidas alcoólicas, tabagismo, refrigerantes e adotar uma dieta balanceada com frutas, legumes e vegetais (ARDENGH; COELHO; OSVALDT, 2008; DAVID et al., 2015). 
De acordo com o relatório da Organização Mundial da Saúde (2018), o câncer é uma das doenças mais mortais do mundo. Aproximadamente, a segunda maior mortalidade é causada pelo câncer. $O$ crescimento descontrolado e a metástase de células anormais são conhecidos como câncer (GAUTAM et al., 2021; CHOUDHURY et al., 2020).

A quercetina (3,5,7-trihidroxi-2- (3,4-di-hidroxifenil) -4Hchromen-4-ona) é um flavonoide natural existente em diferentes alimentos, frutas, vegetais, legumes, (maças, pêra, cebola, espinafre, couve, alface, brócolis, entre outros). Tem efeitos benéficos na saúde humana por meio da mediação de atividades antioxidantes, impactos imunomoduladores e regulação das vias metabólicas (BSIDHU; ZAFAR, 2020; GHAFOURI-FARD et al., 2021). A quercetina tem atraído cada vez mais a atenção devido às suas propriedades farmacológicas: antivirais, antibacterianas, anticarninogênica, antioxidante, efeitos anti-inflamatórios, entre outras. Além disso, a quercetina tem um forte potencial no tratamento de cânceres, pois pode inibir a proliferação de diferentes tipos de células cancerígenas modulando seus processos celulares e impedindo-os de crescer (HIDALGO et al., 2010; INDRAN et al., 2011; HASSAN et al., 2020). Os alimentos ricos em quercetina são uma ótima forma de estimular e fortalecer o sistema imune, pois a quercetina é uma substância antioxidante que elimina os radicais livres do organismo, evitando danos para as células e DNA, podendo prevenir o surgimento de vários problemas de saúde, como, doenças cardíacas, neurodegenerativas, câncer, influencia na inibição de cataratas em diabéticos, anti-histamínicas (antialérgicas) entre outros (GIBELLINI, L., et al., 2010; HU et al., 2017; KATOPODI; ANASTASIA, 2021; PANCHE; DIWAN; CHANDRA, 2016).

\section{METODOLOGIA}

Foi realizada uma revisão sistemática nas bases de dados ScienceDirect, Scielo e Pubmed no mês de setembro de 2021, utilizando os descritores "pancreatic cancer" AND nanoparticles AND quercetin. Foram excluídos artigos que não trouxeram "pancreatic cancer" AND nanoparticles AND quercetin juntos. Assim, foram utilizados 51 artigos. 


\section{RESULTADOS E DISCUSSÕES}

Ao pesquisar nas bases de dados ScienceDirect foram encontrados 531 resultados. Na Scielo 00 resultados e Pubmed 02 resultados. Ao realizar a leitura do título, resumo e palavras-chave foram utilizados para essa revisão 51 artigos.

O câncer é uma das principais causas de morte em todo o mundo. Existem vários obstáculos na terapia do câncer devido aos efeitos colaterais que limitam seu uso (FERLAY et al., 2015). Várias limitações estão associadas às formulações tradicionais, como biocompatibilidade, segurança e toxicidade. Para superar essas limitações, foram introduzidos sistemas de entrega de fármacos à base de lipídios. As nanopartículas lipídicas sólidas (NLS) são vantajosas em termos da prevenção da degradação química dos fármacos e sua flexibilidade em alterar a liberação destes no alvo desejado. NLS têm propriedades únicas, como alta carga de carregamento e proteção dos fármacos do ambiente circundante, maior biodisponibilidade e área de superfície aumentada, possui grande potencial para entrega de fármacos específica ao local e entrega controlada (EZZATI NAZHAD DOLATABADI; VALIZADEH; HAMISHEHKAR, 2015; GAUTAM et al., 2021).

O carcinoma pancreático está entre uma das manifestações comuns de malignidade em nações em desenvolvimento. Os tumores pancreáticos são amplamente divididos em benignos ou maligno de acordo com sua histologia celular (STATHIS; MOORE, 2010; VONDERHEIDE; BAYNE, 2013). Os principais tipos de tumores malignos do pâncreas constituem dois tipos amplos, adenocarcinoma pancreático; que se origina no tecido glandular, correspondendo a $90 \%$ dos casos diagnosticados, a maioria dos casos afeta o lado direito do órgão (a cabeça) e tumor neuroendócrino pancreático; ele se desenvolve a partir de células endócrinas do pâncreas, que passam a produzir hormônios de forma irregular, é responsável por $5 \%$ do total de doenças malignas, é um tipo raro de câncer (STOCK, 2018). Alguns dos fatores de risco constituem excesso de peso, tabagismo, consumo de álcool, exposição ocupacional, fatores dietéticos, entre outros, o que pode dobrar o risco desse desenvolver o câncer (JAMES et al., 2004; CARRIGAN et al., 2007). 
Os sistemas de distribuição de fármacos nanoparticulados foram testados contra o câncer em uma série de estudos científicos. Nos últimos anos, a pesquisa avançada sobre carreadores de lipídios nanoestruturados (CLN) tem atraído considerável atenção devido às vantagens sobre suas contrapartes de primeira geração, nanopartículas lipídicas sólidas (NLS) (BHISE et al., 2017). Recentemente, há um interesse crescente em polifenóis devido à evidência de seu papel promissor na prevenção do câncer (KANDASAMY, 2021). Os polifenóis são produzidos como metabólitos secundários pelas plantas. Seu papel na prevenção do desenvolvimento de tumores por meio de vários mecanismos e redução da massa de células tumorais foi relatado, incluindo a indução de parada do ciclo celular e/ou apoptose. (KAUR; KAUR, 2014; GÜRLER; KIRAZ; BARAN, 2020; AOI; IWASA; MARUNAKA, 2021; BADSHAH et al., 2021).

A formulação de novos sistemas de distribuição de fármacos pode fornecer eficácia aprimorada e/ou toxicidade reduzida para agentes anticâncer (DONG et al., 2021). Pesquisa e desenvolvimento em nanoterápicos de câncer estão progredindo rapidamente e sendo implementada para resolver várias limitações dos sistemas convencionais de entrega de fármacos, como biodistribuição e direcionamento não específicos, baixa biodisponibilidade oral e baixos índices terapêuticos (ARORA; JAGLAN, 2016; ANTAL; ARDELEAN, 2021; NGUYEN et al., 2021). O domínio do câncer à nanoterapêutica está progredindo de forma consistente e sendo usada para remover as várias limitações de métodos convencionais disponíveis para o diagnóstico e tratamento do câncer (SHRIVASTAVA et al., 2021; RASHIDI, 2021). Nanopartículas fornecem uma área interdisciplinar de pesquisa em imagem, diagnóstico e direcionamento do câncer. Com vantagens, como propriedades físicoquímicas avançadas e melhor biodisponibilidade, elas mostram circulação sanguínea prolongada com direcionamento eficiente do tumor (JAVED et al., 2021; QADIR et al., 2021; MIAO et al., 2013; ARANA; GALLEGO; ALKORTA, 2021).

Podem ser utilizados para atingir tecidos específicos em células acessíveis, bem como podem penetrar na barreira hematoencefálica (BHE) devido à adsorção de proteínas do sangue, como as apolipoproteínas, em sua superfície, o que pode facilitar a aderência às células endoteliais (UNER; YENER, 2007; ATTAMA; 
UMEYOR, 2015). Nanopartículas lipídicas com quercetina (NLQ) não apenas reduzem os efeitos colaterais dos quimioterápicos, mas também são capazes de aumentar a citotoxicidade deles nas células cancerosas (GIBELLINI et al., 2011). As NLQ são estudadas para alguns medicamentos anticâncer, pois melhoram os caracteres de solubilidade aquosa e liberação, aumentam a absorção e eficácia celular, efeito inibidor de tumor induzido a apoptose e também são usados como sistemas de distribuição de alvos para alguns órgãos (GIBELLINI et al., 2011; OLIVEIRA; MENDES; TORCHILIN, 2017).

\section{CONCLUSÃO}

A nanotecnologia é uma importante ferramenta à disposição dos pesquisadores, utilizada para superar os problemas da terapia convencional. As NLQ como nanosistemas avançados de fármacos e genes apresentam oportunidades significativas para melhorar a terapêutica médica, por causa do potencial da entrega controlada de fármacos a um tecido-alvo e sua biocompatibilidade, haverá muitos estudos na melhoria da qualidade, eficácia e perfil de segurança dos medicamentos que os usarão no futuro. Soma-se a isso o fato de que apesar dos enormes avanços, os medicamentos disponíveis ainda se mostram pouco eficazes em muitos casos e não seletivos, resultando em danos às células saudáveis e, consequentemente, aos pacientes. Especificamente, este trabalho destaca o uso da quercetina, um composto de origem natural, como um potencial agente antitumoral e assim a NLQ alcançar um lugar importante e promissor no combate ao câncer de pâncreas.

\section{AGRADECIMENTOS}

Gostaria de agradecer ao Programa Institucional de Bolsas de Iniciação Científica (PIBIC-CNPq) e à Universidade Franciscana (UFN) pelo suporte para a realização do presente trabalho. 


\section{REFERÊNCIAS}

ANTAL, D. S.; ARDELEAN, F. Chapter 16 - Challenges in the nanoscale delivery systems development in the pharmaceutical and nutraceutical markets. Mitochondrial Dysfunction and Nanotherapeutics. [s.I.] Academic Press, 2021. p. 441-458.

AOI, W.; IWASA, M.; MARUNAKA, Y. Metabolic functions of flavonoids: From human epidemiology to molecular mechanism. Neuropeptides, v. 88, p. 102163, 1 ago. 2021.

ARANA, L.; GALLEGO, L.; ALKORTA, I. Incorporation of Antibiotics into Solid Lipid Nanoparticles: A Promising Approach to Reduce Antibiotic Resistance Emergence. Nanomaterials (Basel, Switzerland), v. 11, n. 5, p. 1251, 10 maio 2021.

ARDENGH, J. C.; COELHO, N.; OSVALDT, A. B. Pancreatic cancer at an early stage: is it possible to identify it using the scientific and diagnostic instruments currently available? Gastroenterology Archives, v. 45, p. 169-177, jun. 2008.

ARORA, D.; JAGLAN, S. Nanocarriers based delivery of nutraceuticals for cancer prevention and treatment: A review of recent research developments. Trends in Food Science \& Technology, v. 54, p. 114-126, 1 ago. 2016.

ATTAMA, A. A.; UMEYOR, C. E. The use of solid lipid nanoparticles for sustained drug release. Therapeutic Delivery, v. 6, n. 6, p. 669-684, 2015.

BADSHAH, S. L. et al. Antiviral activities of flavonoids. Biomedicine \& Pharmacotherapy, v. 140, p. 111596, 1 ago. 2021.

BHISE, K. et al. Nanostructured lipid carriers employing polyphenols as promising anticancer agents: Quality by design ( $\mathrm{QbD})$ approach. International Journal of Pharmaceutics, v. 526, n. 1, p. 506-515, 30 jun. 2017.

BYUN, J. H.; JUNG, I. H. Phase-specific cancer-immune model considering acquired resistance to therapeutic agents. Applied Mathematics and Computation, v. 391, p. 125555,15 fev. 2021.

CARRIGAN, P. E. et al. Distinctive Heavy Metal Composition of Pancreatic Juice in Patients with Pancreatic Carcinoma. Cancer Epidemiology and Prevention Biomarkers, v. 16, n. 12, p. 2656-2663, 1 dez. 2007.

CHATURVEDI, S.; GARG, A.; VERMA, A. Nano lipid based carriers for lymphatic voyage of anti-cancer drugs: An insight into the in-vitro, ex-vivo, in-situ and in-vivo study models. Journal of Drug Delivery Science and Technology, v. 59, p. 101899, 1 out. 2020. 
CHOUDHURY, $\mathrm{H}$. et al. Advanced nanoscale carrier-based approaches to overcome biopharmaceutical issues associated with anticancer drug 'Etoposide'. Materials Science and Engineering: C, v. 106, p. 110275, 1 jan. 2020.

DAVID, K. I. et al. Dual drug loaded chitosan nanoparticles-sugar-coated arsenal against pancreatic cancer. Colloids and Surfaces B: Biointerfaces, v. 135, p. 689698, nov. 2015.

DONG, S. et al. Emerging role of natural products in cancer immunotherapy. Acta Pharmaceutica Sinica B, 21 ago. 2021.

EZZATI NAZHAD DOLATABADI, J.; VALIZADEH, H.; HAMISHEHKAR, H. Solid Lipid Nanoparticles as Efficient Drug and Gene Delivery Systems: Recent Breakthroughs. Advanced Pharmaceutical Bulletin, v. 5, n. 2, p. 151-159, jun. 2015.

FERLAY, J. et al. Cancer incidence and mortality worldwide: sources, methods and major patterns in GLOBOCAN 2012. International Journal of Cancer, v. 136, n. 5, p. E359-386, 1 mar. 2015.

GAUTAM, L. et al. Emergence of novel targeting systems and conventional therapies for effective cancer treatment. In: Nano Drug Delivery Strategies for the Treatment of Cancers. [s.I.] Elsevier, 2021. p. 1-35.

GHAFOURI-FARD, S. et al. The impact of the phytotherapeutic agent quercetin on expression of genes and activity of signaling pathways. Biomedicine \& Pharmacotherapy, v. 141, p. 111847, set. 2021.

GIBELLINI, L. et al. Quercetin and Cancer Chemoprevention. Evidence-Based Complementary and Alternative Medicine, v. 2011, p. eneq053, 14 abr. 2011.

GONÇALVES, J. O. Smoking as a risk factor for chronic pancreatitis - systematic literature review. 2 mai 2017.

GÜRLER, S. B.; KIRAZ, Y.; BARAN, Y. Chapter 21 - Flavonoids in cancer therapy: current and future trends. In: OZTURK, M.; EGAMBERDIEVA, D.; PEŠIĆ, M. (Eds.). . Biodiversity and Biomedicine. [s.I.] Academic Press, 2020. p. 403-440.

HANI, U. et al. A systematic study of novel drug delivery mechanisms and treatment strategies for pancreatic cancer. Journal of Drug Delivery Science and Technology, v. 63, p. 102539, jun. 2021.

HASSAN, S. et al. Quercetin potentializes the respective cytotoxic activity of gemcitabine or doxorubicin on 3D culture of AsPC-1 or HepG2 cells, through the inhibition of HIF-1 $\alpha$ and MDR1. PLoS ONE, v. 15, n. 10, p. e0240676, 14 out. 2020. 
HIDALGO, M.; SÁNCHEZ-MORENO, C.; DE PASCUAL-TERESA, S. Flavonoidflavonoid interaction and its effect on their antioxidant activity. Food Chemistry, v. 121 , n. 3, p. 691-696, 1 ago. 2010.

HU, B. et al. Food macromolecule based nanodelivery systems for enhancing the bioavailability of polyphenols. Journal of Food and Drug Analysis, Dietary Natural Compounds. v. 25, n. 1, p. 3-15, 1 jan. 2017.

INDRAN, I. R. et al. Recent advances in apoptosis, mitochondria and drug resistance in cancer cells. Biochimica Et Biophysica Acta, v. 1807, n. 6, p. 735-745, jun. 2011.

JAMES, T. A. et al. Risk factors associated with earlier age of onset in familial pancreatic carcinoma. Cancer, v. 101, n. 12, p. 2722-2726, 2004.

JAVED, R. et al. Chapter 3 - Phytonanotechnology: a greener approach for biomedical applications. In: PATRA, C. et al. (Eds.). Biogenic Nanoparticles for Cancer Theranostics. Micro and Nano Technologies. [s.I.] Elsevier, 2021. p. 43-86.

KANDASAMY, G. Chapter 13 - Pharmacokinetics/pharmacodynamics and clinical relationship. In: THORAT, N. D.; KUMAR, N. (Eds.). Nano-Pharmacokinetics and Theranostics. [s.I.] Academic Press, 2021. p. 253-271.

KATOPODI, A.; ANASTASIA, D. Solid Lipid Nanoparticles and Nanostructured Lipid Carriers of natural products as promising systems for their bioactivity enhancement: The case of essential oils and flavonoids. Colloids and Surfaces A: Physicochemical and Engineering Aspects, p. 127529, 14 set. 2021.

KAUR, H.; KAUR, G. A Critical Appraisal of Solubility Enhancement Techniques of Polyphenols. Journal of Pharmaceutics, v. 2014, p. 180845, 2014.

LEE, S.-M. et al. Prevention and treatment of diabetes with resveratrol in a nonobese mouse model of type 1 diabetes. Diabetologia, v. 54, n. 5, p. 1136-1146, maio 2011.

MATHEUS, P. G. Relevance of the pro-oxidant action of quercetin in its mechanism of action as a promising drug in the treatment of cancer. Aleph, p. 81 f., 13 fev. 2017.

MCGUCKIN, E.; CADE, J. E.; HANISON, J. The pancreas. Anaesthesia \& Intensive Care Medicine, v. 21, n. 11, p. 604-610, 1 nov. 2020.

MIAO, J. et al. Drug resistance reversal activity of anticancer drug loaded solid lipid nanoparticles in multi-drug resistant cancer cells. Colloids and Surfaces. B, Biointerfaces, v. 110, p. 74-80, 1 out. 2013.

MOINI, J. Chapter 2 - The Pancreas and Classifications of Insulin. In: MOINI, J. (Ed.). . Epidemiology of Diabetes. [s.I.] Elsevier, 2019. p. 11-23. 
NOBESCHI, L. et al. Diagnosis and prevention of pancreas cancer. Essays and Science: Biological, Agricultural and Health Sciences, v. 16, p. 167-17, 2012.

NGUYEN, T. H. P. et al. Phytochemicals intended for anticancer effects at preclinical levels to clinical practice: Assessment of formulations at nanoscale for non-small cell lung cancer (NSCLC) therapy. Process Biochemistry, v. 104, p. 55-75, 1 maio 2021.

OLIVEIRA, M. S.; MENDES, L. P.; TORCHILIN, V. P. Chapter 18 - Targeted delivery of anticancer drugs: new trends in lipid nanocarriers. In: FICAI, A.; GRUMEZESCU, A. M. (Eds.). Nanostructures for Cancer Therapy. Micro and Nano Technologies. [s.l.] Elsevier, 2017. p. 455-484.

PANCHE, A. N.; DIWAN, A. D.; CHANDRA, S. R. Flavonoids: an overview. Journal of Nutritional Science, v. 5, ed 2016.

QADIR, A. et al. Chapter 3 - Regulatory perspectives of nanomedicines for cancer treatment. In: BEG, S. et al. (Eds.). Nanoformulation Strategies for Cancer Treatment. Micro and Nano Technologies. [s.I.] Elsevier, 2021. p. 29-49.

RASHIDI, L. Different nano-delivery systems for delivery of nutraceuticals. Food Bioscience, v. 43, p. 101258, 1 out. 2021.

SADOUGHI, F.; MANSOURNIA, M. A.; MIRHASHEMI, S. M. The potential role of chitosan-based nanoparticles as drug delivery systems in pancreatic cancer. IUBMB Life, v. 72, n. 5, p. 872-883, maio 2020.

SHRIVASTAVA, N. et al. Chapter 8 - Lipid-based combinational drug delivery systems: Maximizing the performance of cocktail therapy. In: BABOOTA, S.; ALI, J. (Eds.). Nanocarriers for the Delivery of Combination Drugs. Micro and Nano Technologies. [s.I.] Elsevier, 2021. p. 259-305.

SIDHU, J. S.; ZAFAR, T. A. 10 - Indian herbal medicine and their functional components in cancer therapy and prevention. In: KABIR, Y. (Ed.). Functional Foods in Cancer Prevention and Therapy. [s.I.] Academic Press, 2020. p. 169194.

SILVA, E. C. A. et al. Polyphenols Antioxidants of Brazilian Plants Used as Cancer Inhibitors. International Journal of Nutrition. Thieme Revinter Publicações Ltda, set. 2018. Disponível em: <http://www.thiemeconnect.de/DOI/DOI?10.1055/s-0038-1674404>. Acesso em: 16 set. 2021

STATHIS, A.; MOORE, M. J. Advanced pancreatic carcinoma: current treatment and future challenges. Nature Reviews Clinical Oncology, v. 7, n. 3, p. 163-172, mar. 2010. 
STOCK, M. Pancreatic Adenocarcinoma: Pathophysiology and Virotherapy. Pancreatic Adenocarcinoma: Pathophysiology and Virotherapy. May 30th 2018. Disponível em: <https://estudogeral.sib.uc.pt/handle/10316/82121>. Acesso em: 16 set. 2021.

TANG, S.-N. et al. Inhibition of sonic hedgehog pathway and pluripotency maintaining factors regulate human pancreatic cancer stem cell characteristics. International Journal of Cancer, v. 131, n. 1, p. 30-40, 2012.

TRAUB, B.; LINK, K.-H.; KORNMANN, M. Curing pancreatic cancer. Seminars in Cancer Biology, 29 maio 2021.

UNER, M.; YENER, G. Importance of solid lipid nanoparticles (SLN) in various administration routes and future perspectives. International Journal of Nanomedicine, v. 2, n. 3, p. 289-300, 2007.

VONDERHEIDE, R. H.; BAYNE, L. J. Inflammatory networks and immune surveillance of pancreatic carcinoma. Current Opinion in Immunology, Lymphocyte development / Tumour immunology / Cancer immunology: Clinical translation. v. 25, n. 2, p. 200-205, 1 abr. 2013. 